\title{
Sex-Specific Cognitive Deficits and Regional Brain Volume Loss in Mice Exposed to Chronic, Sublethal Hypoxia
}

\author{
WEN-CHUN J. LAN, MATTHEW PRIESTLEY, SONIA R. MAYORAL, LU TIAN, MEHRDAD SHAMLOO, AND ANNA A. PENN
}

Department of Pediatrics [W.J.L., A.A.P.], Stanford University School of Medicine, Stanford, California 94305; Behavioral and Functional Neuroscience Laboratory [M.P., M.S.], Neurosciences Program [S.R.M.], Department of Health Research and Policy-Biostatistics [L.T.], Stanford University, Stanford, California 94305

\begin{abstract}
Male sex is an independent risk factor for long-term neurologic deficits in human preterm infants. Using a chronic, sublethal hypoxia $(\mathrm{CSH})$ mouse model of preterm brain injury, we recently demonstrated acute brain volume loss with an increased male susceptibility to hippocampal volume loss and hypomyelination. We now characterize the long-term, sex-specific effects of CSH on cognition and brain growth. Neonatal mice were treated with $\mathrm{CSH}$ for $8 \mathrm{~d}$, raised in normoxia thereafter and underwent behavioral testing at $6 \mathrm{wk}$ of age. Behavioral assays sensitive to hippocampal function were chosen. $\mathrm{CSH}$-treated males had impairments in associative learning, spatial memory, and long-term social memory compared with control males. In contrast, CSH-treated females were less impaired. Persistent reductions in hippocampal and cerebellar volumes were found in adult CSH-treated males, whereas regional brain volumes in adult $\mathrm{CSH}$-treated females were indistinguishable from controls. Similar to human preterm infants, males exposed to hypoxia are especially vulnerable to short-term and long-term deficits in cognition and brain growth. (Pediatr Res 70: 15-20, 2011)
\end{abstract}

$\mathrm{L}$ ong-term cognitive deficits are common adverse sequelae of preterm birth $(1,2)$. Neuroanatomical differences in regional brain volume and myelination in preterm survivors are increasingly recognized $(3,4)$. Male sex is a strong independent risk factor for adverse cognitive and neuroanatomical outcomes in preterm infants, but the mechanism for their vulnerability is unknown (1). Although rodent models have been developed to study mechanisms of preterm brain injury (5), few studies have looked at males and females separately, limiting our understanding of sex-specific damage.

To elucidate the mechanisms that protect the female developing brain or render males more susceptible to damage, we are exploring sex-specific differences in a rodent model that mimics neuroanatomical findings of preterm brain injury. Although many factors contribute to preterm brain injury, cerebral hypoxia is important because of its frequency in the preterm population from RDS, chronic lung disease, and hypotension (6). A rodent model of chronic, sublethal hypoxia (CSH) has been shown to cause neuroanatomical findings of diffuse brain volume loss,

Received August 5, 2010; accepted January 20, 2011.

Correspondence: Anna A. Penn, M.D., Ph.D., 300 Pasteur Drive, Grant Building S228, Stanford, CA 94305; e-mail: apenn@stanford.edu

Supported by NIH Director's New Innovator Award DP2OD005675, K08 NS044989, John Merck Fund for Developmental Disabilities, Susman \& Asher Foundation, and Packard Children's Health Initiative [A.A.P.]; Lucile Packard Foundation for Children's Health Pediatric Research Fund and Ruth L. Kirschstein National Research Service Award T32-HD007249 [W.J.L.]; and NIMH T32MH020016, NINDS R01NS05389803S1 [S.R.M.]. impaired myelination, and ventriculomegaly, findings that are strikingly similar to those seen in human preterm infants (7). Using this model, we recently demonstrated sex-specific susceptibility to $\mathrm{CSH}$, with males having greater hippocampal volume loss and less myelination than females (8).

We hypothesized that CSH would also lead to sex-specific effects on long-term cognitive development and brain growth, with greater impairment in males. To test this, we selected a panel of behavioral assays to test learning, memory, and social awareness to compare cognitive development in control and CSH-treated mice. Long-term neuroanatomical differences were analyzed at the conclusion of behavioral testing.

\section{MATERIALS AND METHODS}

Hypoxia. All experiments were approved by the Administrative Panel on Laboratory Animal Care at Stanford University (Protocol no. 10829). Hypoxia treatment was carried out as described in Ref. 8. C57BL/6 litters of four male pups, four female pups, a C57BL/6 dam, and a CD-1 foster dam were randomized to hypoxia or normoxia from P3-P11. The hypoxic litters with dams were placed in a chamber where a continuous oxygen sensor and controller unit (Biospherix, NY) adjusted nitrogen gas infusion rates to maintain a fraction of inspired oxygen $\left(\mathrm{FiO}_{2}\right)$ of 0.1 . The normoxic litters remained in ambient air with $\mathrm{FiO}_{2}$ of 0.21 . On P11, the litters in the hypoxia chamber were returned to ambient air. Although there is a significant mortality rate for pups in hypoxia, there is no sex difference in mortality. Litters were weaned at P21.

IntelliCage testing. Automated behavioral testing for the evaluation of learning and memory was conducted in the IntelliCage (NewBehavior AG, Switzerland). Radiofrequency identification devices (RFID; Datamars SA, Switzerland) were implanted s.c. for individual mouse identification. The IntelliCage holds up to 16 mice and provides a group housing environment with unrestricted food access but restricted water access limited to four programmable testing corners. The mice enter testing corners via an opening large enough for only one mouse. Heat sensors in the corners detect mouse entry and the RFID identifies the specific mouse. Each testing corner has two programmable doors that can be open, closed, or openable with a nosepoke to provide water access which is considered reward. A programmable air puff is considered punishment. Above each door are programmable light emitting diode (LED) lights of varying colors for choice discrimination. Corner visits, nosepokes, licks, and visit times were recorded.

Baseline activity level. Testing started at $5 \mathrm{wk}$ of age. Water access was unrestricted in all testing corners. The total visits to testing corners during light and dark cycles were measured over $3 \mathrm{~d}$.

Place learning and reversal testing. Testing started at $6 \mathrm{wk}$ of age. Water access was restricted to two 1-h long drinking sessions per day. During these sessions, each mouse was assigned one specific corner (place learning corner) where water was accessible in response to a nosepoke. The percentage of

Abbreviations: BrdU, 5-bromodeoxyuridine; CSH, chronic, sublethal hypoxia; DMP, delayed matching-to-place; FC, female control; FH, female hypoxia; MC, male control; MH, male hypoxia; MWM, Morris water maze; OEF, ovariectomized female 
nosepokes in each corner was measured for $4 \mathrm{~d}$ (eight sessions). After $4 \mathrm{~d}$, corners were reprogrammed for each mouse so that water became inaccessible in the place learning corner but became accessible in a different corner (reversal corner). After the switch, the percentage of nosepokes in each corner was measured for another $4 \mathrm{~d}$ (eight sessions). Nonparametric permutation test was performed to assess the difference in the probabilities of nosepokes at three sessions after reversal between control and hypoxic animals of both sexes.

Cued punishment testing. Testing started at 9 wk of age. Water access was restricted to two 1-h long drinking sessions per day. During the sessions, in all testing corners, one door was transiently labeled with green LEDs and the other with yellow LEDs. Half of the animals were assigned yellow as correct and green as incorrect; the other half were assigned the opposite. A nosepoke at a door with the incorrect LEDs caused an air puff, whereas a nosepoke at a door with the correct LEDs allowed water access. LED light colors above each door were switched every $30 \mathrm{~min}$ to eliminate place learning as a confounder. The percentage of errors (nosepokes at the incorrect door) was measured for $7 \mathrm{~d}$ (14 sessions). Statistical analysis was performed using a generalized mixed effects regression model to evaluate the difference in error rates between control and hypoxic animals of both sexes. Mousespecific random effect was included to account for within-mice correlations. The regression analysis also adjusted for session variation.

\section{Standard behavioral testing}

Exploratory activity in novel environment. Testing started at $17 \mathrm{wk}$ of age. The Activity Chamber (Med Associates Inc., St. Albans) was used to assess general activity, gross locomotor activity, and exploratory behavior. Assessment took place in a $43.2 \mathrm{~cm} \times 43.2 \mathrm{~cm}$ arena with infrared detectors within a sound-attenuating chamber under dim light. The animal was placed in the center of the arena and allowed to move freely for $10 \mathrm{~min}$ while being tracked by an automated tracking system. Ambulatory time, resting time, and distance moved were recorded.

Morris water maze with delayed matching-to-place. Testing started at $17 \mathrm{wk}$ of age. The delayed matching-to-place (DMP) water maze task was used to assess learning and memory as described previously (9). A darkcolored tank (170 cm in diameter) was filled with opacified water. Each day, each mouse was placed in the Morris water maze (MWM) at one of four randomly selected locations and given up to $90 \mathrm{~s}$ to find a submerged platform. Once the platform was found, the animal was allowed to remain on the platform for $10 \mathrm{~s}$. If the mouse failed to find the platform after $90 \mathrm{~s}$, the animal was placed on the platform for $10 \mathrm{~s}$. Four trials with an intertrial interval of 8-12 min were conducted daily with the platform in a fixed location. Each subsequent day for $6 \mathrm{~d}$, the submerged platform was moved to a new location and the four trials repeated. During each trial, the time to locate the submerged platform (escape latency) was recorded. The mixed effect linear regression analysis was performed in each sex to evaluate the difference in escape latency time savings between trials 1 and 2 on d 2-7 between control and hypoxic animals. Mice and day-specific random effects were included to account for correlations.

Social testing. Testing started at $10 \mathrm{wk}$ of age. Because of the sex-specific design of the sociability assays, only male mice were tested. Mice were individually housed $1 \mathrm{wk}$ before testing. Before social memory testing, randomly selected, individually housed, C57BL/6 ovariectomized female mice (OEF) were put into the home cages of test mice for $4 \mathrm{~h}$ per day for 5-7 $\mathrm{d}$ to reduce sexual behavior. This test was conducted as described in Ref. 10 but modified by adding a sixth trial. The same, never-before-met OEF was placed into the home cage of the test mouse for four 1-min trials with a 10-min intertrial interval. In the fifth trial, a novel OEF was introduced. In the sixth trial, the original OEF from trials 1 to 4 was reintroduced as control. All trials were videotaped and analyzed for socialization time (anogenital, perioral, and body investigation). The mixed effect linear regression model was used to compare socialization time between control and hypoxic animals for the first four sessions. The Wilcoxon test was used for comparisons for the fifth and sixth sessions.

Brain sectioning. Brains were harvested after intracardiac perfusion with $4 \%$ paraformaldehyde. Brains were fixed overnight and dehydrated in $30 \%$ sucrose with $0.01 \%$ sodium azide. Sagittal sections of $50 \mu \mathrm{m}$ thickness were obtained using a sliding microtome. Every fifth section was mounted on a slide.

Volume analysis. Brain sections obtained at $20 \mathrm{wk}$ of age were stained with cresyl violet. Sections were imaged using a Leica DFC280 camera on a Leica stereoscope. Cortex, hippocampus, and cerebellum areas were measured for each section using ImageJ (National Institutes of Health, Bethesda, MD), multiplied by section thickness, and added to determine regional volumes. A linear regression model was used to compare regional brain volumes between control and hypoxic animals. Comparisons were made on absolute volumes within each sex and graphed relative to male normoxic volumes.
Cell proliferation. On $\mathrm{P} 11,5 \mathrm{mg} / \mathrm{kg}$ 5-bromodeoxyuridine (BrdU) was injected intraperitoneally. Brains sections were obtained at $6 \mathrm{wk}$ of age. Immunohistochemistry was done on free-floating sections using 1:1000 antiBrdU (Abcam, Cambridge) and anti-NeuN antibodies (Abcam). Immunostaining was visualized on a Nikon E800 microscope. All BrdU + cells within the dentate gyrus were counted for each section and divided by the dentate gyrus area to calculate BrdU+ cell density. The $t$ test was used to compare the BrdU + cell density between control and hypoxic animals for each sex.

\section{RESULTS}

Ninety-eight mice (57 males and 41 females) were tested in three cohorts (IntelliCage only, IntelliCage + Activity Chamber+MWM/DMP, social testing). In all cohorts, as noted previously $(7,8)$, hypoxic mice weighed significantly less than controls immediately after hypoxia (data not shown). However, as in previous studies, gradual somatic catch-up growth was seen.

Baseline activity. In the IntelliCage, visits to testing corners and circadian patterns of exploratory activity were no different between control and hypoxic animals (Fig. 1A). In the Activity Chamber, no differences were seen in baseline activity as measured by ambulatory duration, resting duration, and distance moved (Fig. $1 B-D$ ). This is consistent with published data reporting greater locomotor activity in young CSHtreated animals that disappeared by 6 wk of age (11).

Place learning and reversal of place learning. Location memory was tested with place learning and reversal tasks in the IntelliCage (Fig. 2). Place learning was assessed by measuring the percentage of nosepokes in the place learning corner over serial drinking sessions. Over the first eight sessions, no difference in place learning was seen between control and hypoxic mice in either sex. All groups showed an increase percentage of nosepokes in the water-accessible place learning corner over time and a corresponding decrease in the water-inaccessible reversal corner. After water access was changed to the reversal corner after session 8, all groups showed a decline in the percentage of nosepokes in the water-inaccessible place learning corner and a corresponding increase in the water-accessible reversal corner. However, reversal of place learning was significantly slower in hypoxic
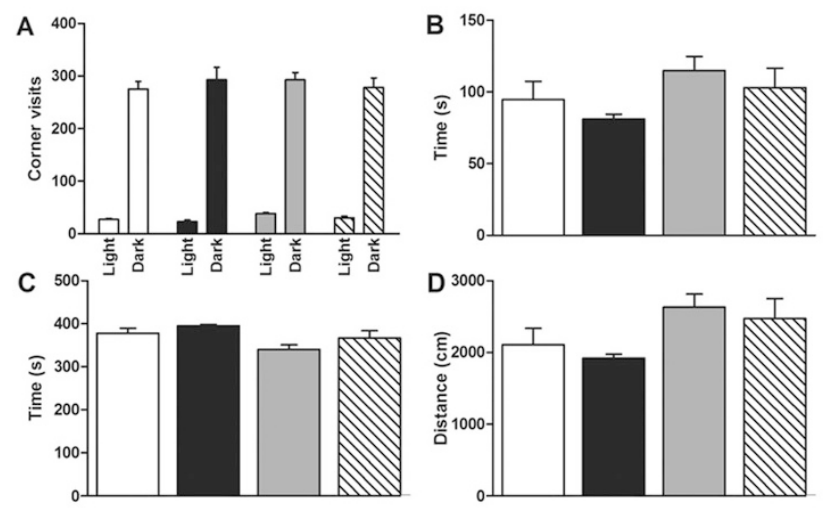

Figure 1. Activity levels. (A) Hypoxia does not affect the number of IntelliCage corner visits in light and dark cycle, $n=28(\mathrm{MC}=7, \mathrm{MH}=7, \mathrm{FC}=$ $8, \mathrm{FH}=6) .(B-D)$ Hypoxia does not affect ambulatory duration, resting duration, or distance moved in the Activity Chamber, $n=28(\mathrm{MC}=4$, $\mathrm{MH}=10, \mathrm{FC}=5, \mathrm{FH}=9)$. White $=\mathrm{MC}$, black $=\mathrm{MH}$, gray $=\mathrm{FC}$, hatched $=\mathrm{FH}$. 

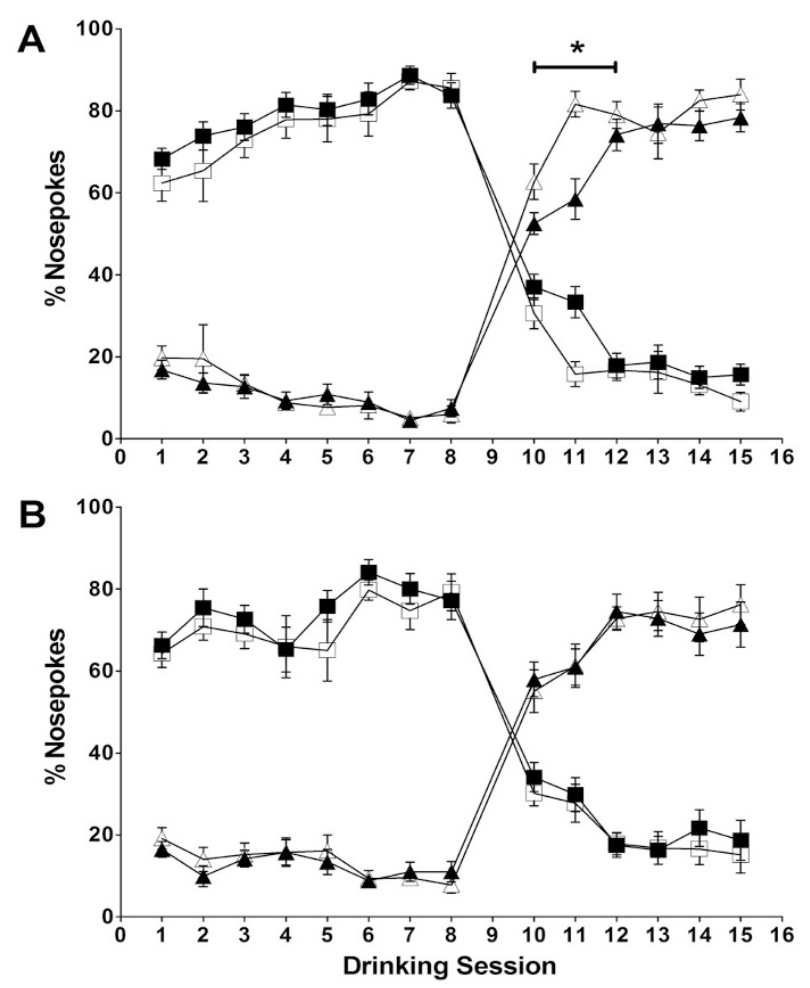

Figure 2. IntelliCage place learning and reversal. $(A)$ In males, hypoxia does not affect place learning but delays reversal of place learning. $(B)$ In females, hypoxia does not affect place learning or reversal of place learning, $n=57$ $(\mathrm{MC}=12, \mathrm{MH}=17, \mathrm{FC}=13, \mathrm{FH}=15) . \square$, normoxia/place learning corner; $\mathbf{\square}$, hypoxia/place learning corner; $\triangle$, normoxia/reversal corner; $\boldsymbol{\Delta}$, hypoxia/reversal corner. ${ }^{*} p<0.05$.

males than controls (Fig. 2A, sessions 10-11). No delay in reversal of place learning was seen in hypoxic females compared with controls (Fig. 2B). Eventually, both hypoxic and control males achieved the same percentage of nosepokes at the reversal corner, implying a deficit in the speed of reversal of place learning but not in the eventual ability to learn the reversed location. Impairment in reversal of place learning is considered to be a sensitive test for hippocampal dysfunction $(12,13)$. Deficits with place learning can be seen with major neurotoxic (14), traumatic (15), or ischemic (16) hippocampal lesions but were not detected in this model.

Cued punishment. Associative learning was tested with the cued punishment task in the IntelliCage (Fig. 3). Associative learning was considered impaired in animals with greater percentages of punished, errant nosepokes. Error rates in both hypoxic and control mice decreased to less than chance by the first session. However, hypoxic males consistently made a higher percentage of errant nosepokes (Fig. $3 A$ ), with an OR of $1.89(p<0.05)$. Hypoxic females also seemed to make more errant nosepokes compared with controls (Fig. $3 B$ ) but were less significantly affected than males, with an OR of 1.5 $(p=0.14)$.

Morris water maze. Spatial learning and working spatial memory were tested with the MWM (Fig. 4). The MWM is a well-validated method to assess spatial memory (12). In the DMP variation of the MWM, the submerged platform is relocated every day, specifically testing working spatial mem-
A

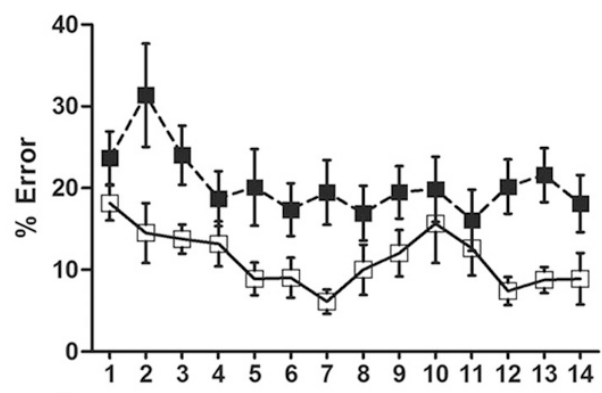

B

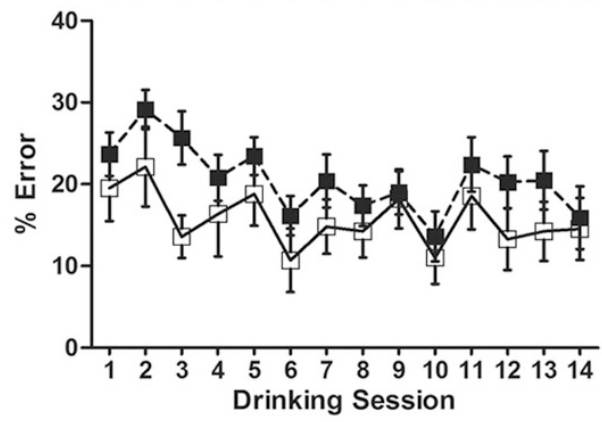

Figure 3. Cued punishment. (A) Males exposed to hypoxia make a significantly higher percentage of errant nosepokes than controls (OR, 1.89; 95\% CI, [1.05-3.39]; $p<0.05)$. (B) Females exposed to hypoxia are less affected than males (OR, 1.50; 95\% CI, [0.89-2.55]; $p=0.14), n=56(\mathrm{MC}=11, \mathrm{MH}=$ $17, \mathrm{FC}=13, \mathrm{FH}=15$ ). $\square$, normoxia; $\square$, hypoxia.
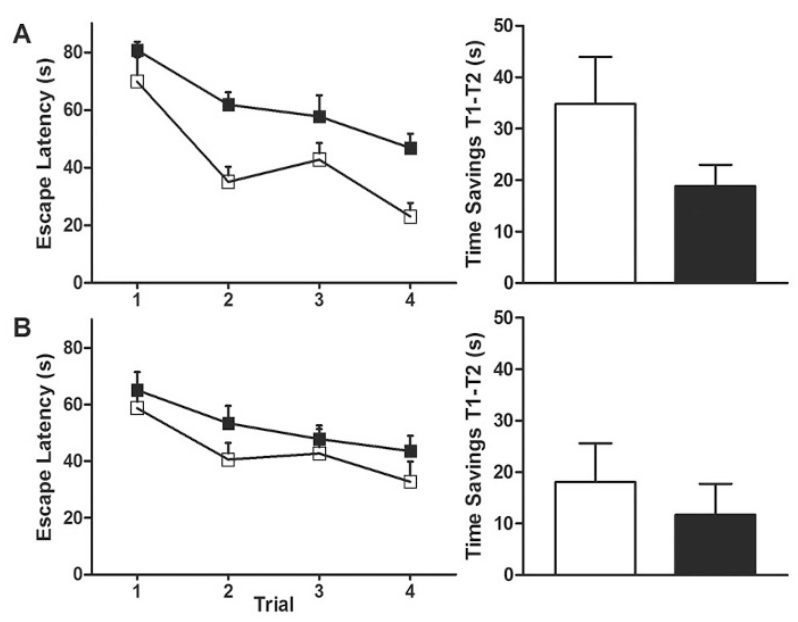

Figure 4. Morris water maze. (A) Males exposed to hypoxia have longer escape latencies in subsequent trials and less time savings between trial 1 and trial 2 than controls (18.9 s vs. $34.8 \mathrm{~s}, p=0.09)$. (B) Females exposed to hypoxia are less affected than males (11.7 s vs. $18.1 \mathrm{~s}, p=0.50), n=28$ $(\mathrm{MC}=4, \mathrm{MH}=10, \mathrm{FC}=5, \mathrm{FH}=9) . \square$, normoxia; $\mathbf{\square}$, hypoxia.

ory by measuring time savings from trial 1 to 2 over multiple days and platform locations. In the first trial, there was no difference in escape latency between control and hypoxic animals as expected. However, hypoxic males had longer escape latencies in subsequent trials, suggesting spatial learning deficits (Fig. 4A, left). Time savings between trials 1 and 2 (Fig. $4 A$, right) were greater in controls compared with hypoxic males, approaching statistical significance. Hypoxic females seemed to have slightly longer escape latencies in subsequent trials compared with controls, although the effect was less pronounced (Fig. 4B, left). Time savings between 
trials 1 and 2 (Fig. 4B, right) were not significantly greater in controls compared with hypoxic females.

Social testing. Sociability, social recognition, preference for social novelty, and social memory were assessed using a modified five-trial social memory test (Fig. 5). A high socialization time was seen in both groups in trial 1, implying that sociability was unaffected. When the same OEF was reintroduced in trials 2-4, socialization time declined in both groups, implying intact social recognition and memory. When a novel OEF was introduced in trial 5, socialization time increased in both groups, implying intact preference for social novelty. With reintroduction of the original OEF, a decrease in socialization time was seen in both groups. However, hypoxic males

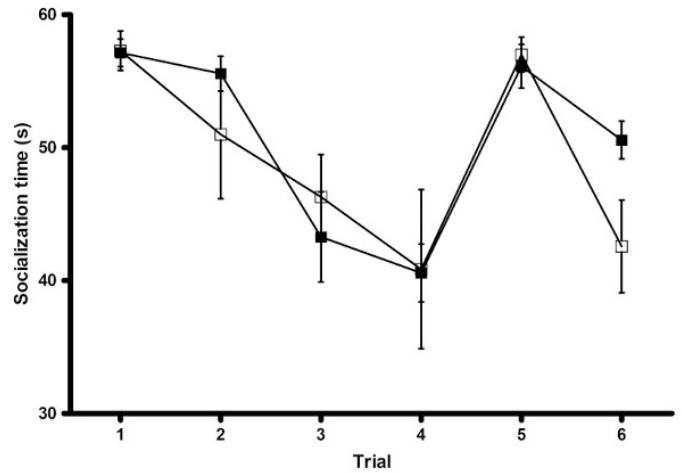

Figure 5. Social testing. The standard pattern of high socialization time (trial 1) followed by decreased socialization time (trials $2-4$ ) and increased socialization time with a novel OEF (trial 5) demonstrates intact short-term social memory and preference for social novelty in both groups. Reintroduction of the original $\mathrm{OEF}$ in trial 6 reveals that hypoxic males have less decline in socialization time than controls, suggesting impairment of long-term social memory ( $5.5 \mathrm{~s} v s .14 .4 \mathrm{~s}, p=0.08), n=14(\mathrm{MC}=7, \mathrm{MH}=7)$. $\square$, normoxia; $\mathbf{\square}$, hypoxia. had a lesser decline in socialization time $(5.5 \mathrm{~s}$ versus $14.4 \mathrm{~s}$, $p=0.08$ ), suggesting long-term social memory impairment.

Brain volume analysis. Regional brain volumes (cerebrum, cerebellum, and hippocampus) were measured in a subset of mice after behavioral testing. In our previous work (8), mice exposed to hypoxia experienced a $31 \%$ reduction in cortical volume and a $40 \%$ reduction in cerebellar and hippocampal volume at P11. In particular, sex-specific differential volume loss in the hippocampus was seen $(43 \%$ reduction in males, $36 \%$ reduction in females). By 20 wk of age, cortical volumes in hypoxic males and females recovered completely compared with sex-matched controls (Fig. 6A). However, hippocampal volumes remained $25 \%$ smaller $(p<0.01)$ and cerebellar volumes remained $23 \%(p<0.01)$ smaller in hypoxic males compared with controls (Fig. $6 B$ and $C$ ). In contrast, hypoxic females recovered hippocampal and cerebellar volumes to control levels.

Cell proliferation. In a separate group of mice, BrdU was injected intraperitoneally on P11 to assess the effect of hypoxia on cell proliferation in the hippocampus. Proliferation in the hippocampus was decreased in the dentate gyrus after CSH (Fig. 6D). BrdU+ cell density is reduced in hypoxic males compared with controls, approaching statistical significance $(p=0.08)$. BrdU + cell density also seems slightly decreased in hypoxic females compared with controls, although this was not statistically significant $(p=0.45)$.

\section{DISCUSSION}

The CSH rodent model closely mimics preterm brain injury. In preterm infants, chronic, low-grade cerebral hypoxia has been correlated with adverse neurodevelopmental outcomes (17). This cerebral hypoxia is simulated by exposing mice pups to $\mathrm{CSH}$ from $\mathrm{P} 3$ to $\mathrm{P} 11$, a period of mouse brain
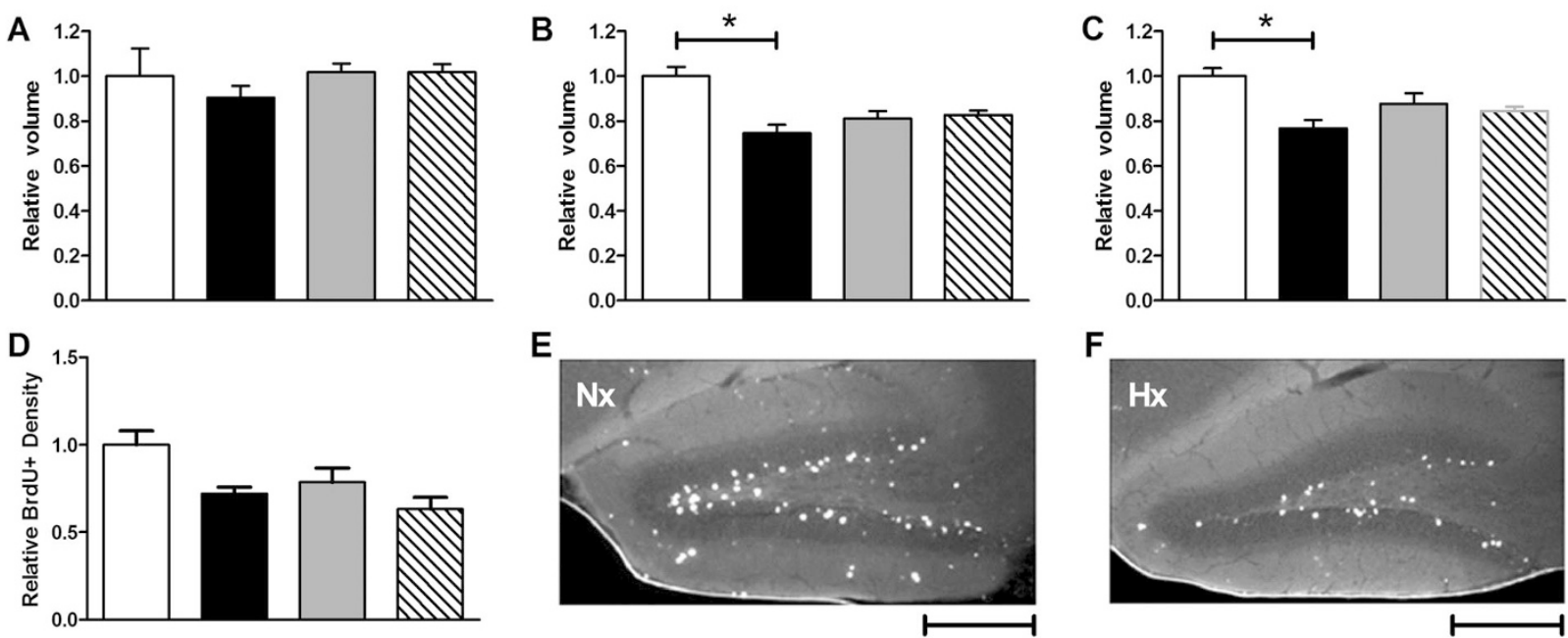

Figure 6. Brain section analysis. (A) Cortical volumes in hypoxic animals have recovered to control levels. (B) Hippocampal volumes in adult hypoxic males remain significantly smaller than controls $(\mathrm{MH} 0.75 \mathrm{vs}$. MC $1, p<0.01)$ while hippocampal volumes in hypoxic females recover. $(C)$ Cerebellar volumes in adult hypoxic males remain significantly smaller than controls (MH $0.77 v s$. MC $1, p<0.01)$ while cerebellar volumes in hypoxic females have recovered, $n=$ $21(\mathrm{MC}=4, \mathrm{MH}=7, \mathrm{FC}=4, \mathrm{FH}=6) .(D) \mathrm{BrdU}+$ cell density in the dentate gyrus of hypoxic males is reduced compared with controls $(p=0.08)$. BrdU+ cell density in the dentate gyrus of hypoxic females trends toward reduction compared with controls but is not significant $(p=0.45)$. $(E$ and $F)$ BrdU immunostaining in the dentate gyrus of normoxic $(\mathrm{Nx})$ and hypoxic $(\mathrm{Hx})$ male hippocampus at $\times 4$. Scale bar $=500 \mu \mathrm{m}, n=16(\mathrm{MC}=3, \mathrm{MH}=5, \mathrm{FC}=$ $3, \mathrm{FH}=5$ ). Volumes and cell densities normalized to male controls. White $=\mathrm{MC}$, black $=\mathrm{MH}$, gray $=\mathrm{FC}$, hatched $=\mathrm{FH}$. 
development matching the third trimester in humans. Brain volume loss, reduced subcortical white matter, ventriculomegaly (7), and disorganization of white matter tracts (11) are seen in both CSH-treated rodents and in preterm humans. Male mice exposed to CSH were particularly susceptible to hippocampal volume loss and hypomyelination when measured immediately after hypoxia (8). Here, we present the first study on long-term, sex-specific effects of CSH on neurobehavioral outcomes and brain volume recovery.

The behavioral testing performed was prompted by our observation of greater hippocampal volume loss in males after CSH (8). The role of the hippocampus in associative learning is increasingly appreciated (18), and its role in spatial memory and spatial information processing is well established $(12,19)$. Our behavioral assays were selected specifically to test these functions. We used the IntelliCage, a recently developed behavioral screening tool that has been used to detect cognitive impairments related to hippocampal damage (20). With the IntelliCage, programmable delivery of cues, reward, and punishment combined with automated tracking of individual mice allows for multiple behavioral assays to be done on a large cohort of mice simultaneously. Environmental conditions affect standard behavioral testing (21), but the IntelliCage requires minimal handling and training. Mice live in a natural, enriched, social environment, reducing anomalous behaviors related to individual housing needed for certain tests (22). Automated data collection increases throughput, reducing the time and cost of behavioral screening. Using the IntelliCage, we detected greater long-term impairments in reversal of place learning and associative learning in males, suggesting that males subjected to CSH are more susceptible not only to short-term hippocampal injury but also to longterm hippocampal dysfunction.

Although the IntelliCage has been used in several studies $(20,23)$, its use in this model is novel. To validate the IntelliCage results, we performed MWM testing, a standard behavioral assay for hippocampus-dependent spatial learning and memory. Consistent with IntelliCage results, greater deficits were again seen in males exposed to CSH on the MWM.

Social testing was included because mounting evidence suggests that preterm infants, particularly males, are at risk for socialization abnormalities $(2,24)$. Sociability, social recognition, and short-term social memory were unaffected. The lack of a difference in most measures of social testing may reflect an inability of our assay to detect subtle social deficits, particularly social deficits that are often subjective in human studies (25). Most rodent studies demonstrating significant social deficits have involved knockout mice $(10,26)$, extreme models whose phenotypes may not be replicated by our model. Long-term social memory seemed impaired in hypoxic males consistent with hippocampal dysfunction.

In this study, long-term hippocampal and cerebellar volume loss persisted only in CSH-treated male. Cell proliferation in the hippocampus was attenuated in CSH-treated males, which may explain in part their inability to recover hippocampal volume. Our data suggest a link between decreased hippocampal size and impaired long-term hippocampal-dependent cognitive function. However, IntelliCage and MWM testing can be affected by changes in brain regions beyond the hippocampus. Volumetric analysis suggests that cerebellar damage may also be involved in the persistent male deficits. Cortical damage is less likely to contribute because cortical volumes recover by adulthood, although recovery of volumes may not equate to recovery of function. This cortical volume recovery is consistent with data describing catch-up cortical growth (7) and enhanced cortical neurogenesis (27) after CSH. Previous work showed a brisk proliferative response to $\mathrm{CSH}$ in the cortical subventricular zone (27), in contrast to our observation of decreased proliferation in the post-CSH hippocampus. Regional differences in cell proliferation in CSH-treated males may explain why cortical volumes recover while hippocampal volumes remain reduced in adulthood. The inability of males to completely recover from cerebellar volume loss was unexpected; no sex differences were seen in cerebellar volume loss in our previous study (8). From observation, hypoxic males and females have erratic, jittery movements shortly after hypoxia. These movements become less noticeable over time and are not observed by adulthood, suggesting recovery of gross cerebellar function, if not cerebellar volume.

It is interesting that in very LBW human infants imaged by MRI at $15 \mathrm{y}$ of age, males had less hippocampal and cerebellar white matter than females (28), similar to our mice. Strikingly, in that same study, hippocampal and cerebellar white matter volumes were correlated with cognitive and perceptual functions. Although we did not observe long-term differences in myelination using histological methods (data not shown), other work has reported DTI abnormalities in adult male mice exposed to CSH (11).

Behavioral studies are often performed on male mice only because potential effects of estrus on behavior are unknown. However, testing for sex differences necessitates study of both sexes. By cohousing females that suppresses estrus cycles (29) and comparing hypoxic with control females, we reduced the confounding effects of estrous. The neglect of sex-specific analysis in neuroscience research has recently been criticized (30). Our results demonstrate the need to study the effects of brain injury in a sex-specific manner to identify male risk factors or female protective factors that could lead to targeted therapeutic modalities. Our study demonstrates the relative ease and importance of sex comparisons.

Although sex differences are noted in many diseases and model systems, the mechanism underlying the differences remains unclear. Sex differences may be related to different sex steroid exposure or receptor expression between males and females or to differential temporal development or the male versus female brain, thus shifting the timing of susceptibility (31). Finally, sex differences may be due to yetunidentified genetic differences from sex chromosomes (32).

We have established the viability of the CSH mouse model for sex-specific neurodevelopmental outcome studies. Furthermore, we have demonstrated the utility of novel (IntelliCage) and standard (MWM) behavioral assays as methods to test the effects of preterm brain injury in animal models. This model can be used to develop and test potential neuroprotective strategies tailored to the sex-specific requirements of vulnerable preterm infants. 
Acknowledgments. We thank Ghezal Omar and Jonathan Tran for their histology assistance.

\section{REFERENCES}

1. Hintz SR, Kendrick DE, Vohr BR, Kenneth Poole W, Higgins RD, For The Nich Neonatal Research Network 2006 Gender differences in neurodevelopmental outcomes among extremely preterm, extremely-low-birthweight infants. Acta Paediatr 95:1239-1248

2. Charkaluk ML, Truffert P, Fily A, Ancel PY, Pierrat V 2010 Neurodevelopment of children born very preterm and free of severe disabilities: the Nord-Pas de Calais Epipage cohort study. Acta Paediatr 99:684-689

3. Peterson BS, Vohr B, Staib LH, Cannistraci CJ, Dolberg A, Schneider KC, Katz KH Westerveld M, Sparrow S, Anderson AW, Duncan CC, Makuch RW, Gore JC, Ment LR 2000 Regional brain volume abnormalities and long-term cognitive outcome in preterm infants. JAMA 284:1939-1947

4. Thompson DK, Warfield SK, Carlin JB, Pavlovic M, Wang HX, Bear M, Kean MJ Doyle LW, Egan GF, Inder TE 2007 Perinatal risk factors altering regional brain structure in the preterm infant. Brain 130:667-677

5. Yager JY, Ashwal S 2009 Animal models of perinatal hypoxic-ischemic brain damage. Pediatr Neurol 40:156-167

6. Back SA, Rivkees SA 2004 Emerging concepts in periventricular white matter injury. Semin Perinatol 28:405-414

7. Ment LR, Schwartz M, Makuch RW, Stewart WB 1998 Association of chronic sublethal hypoxia with ventriculomegaly in the developing rat brain. Brain Res Dev Brain Res 111:197-203

8. Mayoral SR, Omar G, Penn AA 2009 Sex differences in a hypoxia model of preterm brain damage. Pediatr Res 66:248-253

9. Steele RJ, Morris RG 1999 Delay-dependent impairment of a matching-to-place task with chronic and intrahippocampal infusion of the NMDA-antagonist D-AP5. Hippocampus 9:118-136

10. Ferguson JN, Young LJ, Hearn EF, Matzuk MM, Insel TR, Winslow JT 2000 Social amnesia in mice lacking the oxytocin gene. Nat Genet 25:284-288

11. Chahboune H, Ment LR, Stewart WB, Rothman DL, Vaccarino FM, Hyder F, Schwartz ML 2009 Hypoxic injury during neonatal development in murine brain: correlation between in vivo DTI findings and behavioral assessment. Cereb Cortex 19:2891-2901

12. Vorhees CV, Williams MT 2006 Morris water maze: procedures for assessing spatial and related forms of learning and memory. Nat Protoc 1:848-858

13. Bannerman DM, Deacon RM, Seeburg PH, Rawlins JN 2003 GluR-A-Deficient mice display normal acquisition of a hippocampus-dependent spatial reference memory task but are impaired during spatial reversal. Behav Neurosci 117:866-870
14. McNamara RK, Skelton RW 1993 The neuropharmacological and neurochemical basis of place learning in the Morris water maze. Brain Res Brain Res Rev 18:33-49

15. Logue SF, Paylor R, Wehner JM 1997 Hippocampal lesions cause learning deficits in inbred mice in the Morris water maze and conditioned-fear task. Behav Neurosci 111:104-113

16. Auer RN, Jensen ML, Whishaw IQ 1989 Neurobehavioral deficit due to ischemic brain damage limited to half of the CA1 sector of the hippocampus. J Neurosci 9:1641-1647

17. Pryds O 1994 Low neonatal cerebral oxygen delivery is associated with brain injury in preterm infants. Acta Paediatr 83:1233-1236

18. Brasted PJ, Bussey TJ, Murray EA, Wise SP 2003 Role of the hippocampal system in associative learning beyond the spatial domain. Brain 126:1202-1223

19. Morris RG, Garrud P, Rawlins JN, O'Keefe J 1982 Place navigation impaired in rats with hippocampal lesions. Nature 297:681-683

20. Voikar V, Colacicco G, Gruber O, Vannoni E, Lipp HP, Wolfer DP 2010 Conditioned response suppression in the IntelliCage: assessment of mouse strain differences and effects of hippocampal and striatal lesions on acquisition and retention of memory. Behav Brain Res 213:304-312

21. Crabbe JC, Wahlsten D, Dudek BC 1999 Genetics of mouse behavior: interactions with laboratory environment. Science 284:1670-1672

22. Wolfer DP, Litvin O, Morf S, Nitsch RM, Lipp HP, Wurbel H 2004 Laboratory animal welfare: cage enrichment and mouse behaviour. Nature 432:821-822

23. Mechan AO, Wyss A, Rieger H, Mohajeri MH 2009 A comparison of learning and memory characteristics of young and middle-aged wild-type mice in the IntelliCage. J Neurosci Methods 180:43-51

24. Johnson S, Hollis C, Kochhar P, Hennessy E, Wolke D, Marlow N 2010 Autism spectrum disorders in extremely preterm children. J Pediatr 156:525-531

25. Schmidt LA, Miskovic V, Boyle MH, Saigal S 2008 Shyness and timidity in young adults who were born at extremely low birth weight. Pediatrics 122:e181-e187

26. Bielsky IF, Hu SB, Ren X, Terwilliger EF, Young LJ 2005 The V1a vasopressin receptor is necessary and sufficient for normal social recognition: a gene replacement study. Neuron 47:503-513

27. Fagel DM, Ganat Y, Silbereis J, Ebbitt T, Stewart W, Zhang H, Ment LR, Vaccarino FM 2006 Cortical neurogenesis enhanced by chronic perinatal hypoxia. Exp Neurol 199:77-91

28. Martinussen M, Flanders DW, Fischl B, Busa E, Lohaugen GC, Skranes J, Vangberg TR, Brubakk AM, Haraldseth O, Dale AM 2009 Segmental brain volumes and cognitive and perceptual correlates in 15-year-old adolescents with low birth weight. J Pediatr 155:848-853

29. McKinney TD 1972 Estrous cycle in house mice: effects of grouping, preputial gland odors, and handling. J Mammal 53:391-393

30. 2010 Putting gender on the agenda. Nature 465:665

31. McCarthy MM 2009 The two faces of estradiol. Neuroscientist 15:599-610

32. Mage DT, Donner M 2006 Female resistance to hypoxia: does it explain the sex difference in mortality rates? J Womens Health (Larchmt) 15:786-794 